Fecha de recepción: abril 2020 Fecha de aceptación: mayo 2020 Versión final: junio 2020

\section{Bases y fundamentos para la detección de usuarios tipificados en el Diseño de Comportamiento sostenible del consumidor}

Elizabeth Retamozo ${ }^{(1)}$, Greta Clinckspoor ${ }^{(2)}$ y

Carolina Panzone ${ }^{(3)}$

\begin{abstract}
Resumen: Los artefactos se entienden como poseedores de cualidades intrínsecas a su proceso proyectual, que determinan la manera en la que los usuarios los perciben, aprecian, y emplean. Para que el diseño ambientalmente sostenible se consolide, debe considerarse la evaluación de las reacciones de los sujetos ante diferentes motivaciones.

El objetivo de este ensayo es fundamentar, mediante términos teórico-metodológicos, las definiciones conceptuales de los usuarios tipificados en el Diseño de Comportamiento Sostenible, estudiados por autores especializados en psicología ambiental que conciben la conducta desde de diferentes dimensiones, para identificar los distintos perfiles ambientales. Finalmente, el trabajo entreabre la profundización del objetivo hacia un futuro estudio, en el cual se aborde la problemática en el contexto de situaciones extraordinarias, como una pandemia a nivel mundial.
\end{abstract}

Palabras clave: Diseño sustentable - Diseño conductual - Hábitos sustentables - Psicología ambiental - Tipos de usuarios.

[Resúmenes en inglés y portugués en la página 121]

(1) Diseñadora Industrial (UNMDP). Doctoranda (UBA). Becaria Doctoral (CONICET). Integrante del Grupo de Investigación de Diseño Sustentable (GIDSU), del Centro de Investigaciones Proyectuales y Acciones de Diseño Industrial (CIPADI).

(2) Diseñadora Industrial (UNMdP). Docente en Sociología de Diseño Industrial (UNMdP). Especialista en Gestión Ambiental Metropolitana (FADU-UBA). Becaria CONICET. Doctorado (UNLu). Integrante del Instituto del Hábitat y el Ambiente de FAUDUNMdP.

(3) Diseñadora Industrial (UNLP). Doctoranda (UBA). Becaria Doctoral (CIC). Integrante del Grupo de Investigación de Diseño Sustentable (GIDSU), del Centro de Investigaciones Proyectuales y Acciones de Diseño Industrial (CIPADI). 


\section{Introducción}

El sistema económico actual basado en la máxima producción, el consumo, la explotación ilimitada de recursos y el beneficio como única articulación virtuosa hacia la prosperidad económica resulta insostenible a largo plazo (Martínez Alier, 2009; Latouche, 2012). Lo cual se entiende desde la visión antropocéntrica, que ha concebido al ser humano por encima del resto de los seres vivos y sus necesidades por sobre la supervivencia de los ecosistemas. En este sentido se entiende que la transición hacia la sostenibilidad demanda cambios radicales en la forma que producimos, consumimos y, consecuentemente, en el modo en el cual vivimos (Manzini \& Meroni, 2007).

Desde su campo de acción, el diseño industrial, en todas sus variantes, tiene una gran posibilidad de contribuir en la reducción de los impactos negativos sobre el ambiente. Se estima que, por encima del $80 \%$ del impacto ambiental relacionado con los productos se determina durante la etapa de diseño (Environmental Change Institute, 2005). A modo de respuestas y soluciones, algunas corrientes de diseño se han planteado como objetivo final disminuir el consumo de recursos naturales, orientadas hacia diferentes fases del ciclo de vida del producto, entre ellas: el Ecodiseño, el Diseño Sustentable y el Diseño Conductual, entre otras. En este sentido se define al Behavioural Design o Diseño de Comportamiento (DC), enfocada en la etapa de uso, como un campo emergente que combina teorías y métodos para provocar cambios en los comportamientos que causan impactos negativos, tales como derroche de recursos. En otras palabras, es una rama del diseño que puede emplearse para influir en las costumbres humanas (Lockton, 2010; Niedderer, 2014).

Bajo esta perspectiva, surge un Proyecto de Investigación; radicado en el Centro de Investigaciones Proyectuales y Acciones de Diseño Industrial (CIPADI), de la Facultad de Arquitectura, Urbanismo y Diseño de la Universidad Nacional de Mar del Plata; denominado Diseño con intención, motivación de conductas sustentables (2019-2020), actualmente en curso. El mismo, tiene por objetivo desarrollar un modelo de estrategias de DC, tendiente a promover conductas sustentables en torno a los hábitos vinculados con la disposición de residuos que se generan dentro de la propia Universidad. Para realizar esta propuesta, fue necesario desarrollar una metodología de trabajo que permitiera conocer a los usuarios del contexto de estudio, es decir, determinar cuál es su perfil de comportamiento, entendiendo este concepto como aquellas particularidades psicológicas que poseen los mismos. El principal problema detectado fue que algunos enfoques estudiados del DC, se encausan en diseñar según una tipología de usuario pero no explicitan de qué manera el diseñador puede determinar su clasificación. El enfoque seleccionado para continuar con esta investigación fue el utilizado por la diseñadora Olaya Rodríguez (2018). En pos de llevar a la práctica el proceso de tipificación que desarrolla la autora antes mencionada resultó necesario indagar en torno a las siguientes preguntas: ¿Cómo identificar a los distintos usuarios?, ¿Qué parámetros se deben utilizar para reconocerlos? y finalmente, ¿Cómo medir sus conductas para establecer qué tipo de usuario resultan? A partir de estas incógnitas, se optó por comenzar con un paso previo que permita mejorar el entendimiento de la tipificación de usuarios para establecer el tipo de intervención de diseño más adecuado. Este aspecto es compartido habitualmente en diversos proyectos e investigaciones de diseño, en conjunto con la falta de instrumentos disponibles para la recolección de datos. 
De esta manera, al haber detectado el vacío explícito de conocimiento en este campo, esta investigación plantea su objetivo principal en dirección a aportar fundamentos teóricometodológicos que permitan detectar a los usuarios que tipifica el Diseño de Comportamiento sostenible. Para cumplir con el objetivo, se realiza la revisión de los principales antecedentes y el cruzamiento de las herramientas principales de medición de comportamiento, utilizadas por los autores dedicados a la psicología de conducta ambiental, como el uso de las sub-escalas de conducta sustentable y la tipificación de usuarios propuesta por Olaya Rodríguez (2018). Asimismo, en relación a la situación mundial de pandemia, se considera que si bien la revisión conceptual que plantea el artículo se consolida en contextos evolutivos pero habituales, debe precisarse que muchos parámetros en torno al diseño de comportamientos sustentables se ven alterados con nuevas variables.

El siguiente estudio se estructura en cuatro apartados y concluye con las reflexiones finales. En el Punto 1, subtitulado El Diseño Conductual, revisión de los principales referentes y estado actual, se reconocen y contrastan las metodologías y los modelos de dicha corriente. En el punto 2, La psicología de la Conducta Sustentable, se interpretan los conceptos que posteriormente se vinculan con la categorización de usuarios que realiza Olaya Rodríguez (2018); los cuales se profundizan en el apartado siguiente. Por último, en el Punto 3, Determinación de las relaciones existentes en la categorización que realiza Olaya Rodríguez (2018) y las dimensiones de la conducta sustentables, se trabaja con el cruce de los datos desarrollados en los dos primeros puntos y permite dar cuenta de cómo se establece el recorrido realizado para detectar al tipo de usuario, ya tipificado, hacia el cual se diseña.

\section{El Diseño Conductual, revisión de los principales referentes y estado actual}

El DC entiende al diseño como una herramienta para influir positivamente en el comportamiento de las personas. Esta forma de interpretar la relación dialéctica entre el diseño y las personas ha sido abordada por varios autores. Por un lado, Norman (1988), desglosa y describe los factores que intervienen en el proceso de uso de los objetos. En éste libro el autor establece que el comportamiento de una persona en interacción con un artefacto es el resultado de lo que permite el objeto y las restricciones que presenta. Norman introduce por primera vez la palabra affordance al ámbito del diseño, para referirse a las posibilidades de acción que presenta un producto ante el usuario; según el autor, son "las propiedades percibidas y reales del objeto, principalmente aquellas propiedades fundamentales que determinan cómo se podría usar" (Norman, 1988, p. 21). A su vez, desarrolla el concepto de constraints, como las características que proporcionan las "cuatro tipos diferentes de limitaciones: físicas, semánticas, culturales y lógicas” (p. 110). Estas hacen más comprensible las posibilidades de uso presentes en el elemento, ya que guían la acción de diversas maneras. Estos aportes, entre otros, fueron considerados una base sustancial para estudios posteriores. Un factor de relevancia en sus estudios es la comprensión de la percepción como un elemento decisivo en la interacción con los objetos. Así también, la noción del diseño como dispositivo de comunicación entre el objeto y el usuario, y la forma en que permite optimizar ese conducto comunicativo para realizar la experiencia de su uso. 
En 1992, Akrich explica que la relación entre el usuario y el objeto depende en gran parte del guión de uso proyectado por el diseñador y por su articulación con la percepción de quien lo utiliza. A partir de esta nueva perspectiva, se actualiza la línea teórica al incorporar la variable tecnológica y el desarrollo del diseño desde la maquinaria, ya que muchos de los ejemplos trabajados por los autores que lo anteceden no reflejan su análisis sobre los avances más modernos de la época.

En 2002, Fogg explica cómo cambiar actitudes o comportamientos en el campo de la tecnología, tópico que hasta el momento no se había explicitado. A lo largo de su trabajo, ha desarrollado varios aportes de los que se ha nutrido el DC. Uno de ellos ha sido el Fogg Behavioural Model (2007), un modelo psicológico que expone los elementos necesarios para influenciar el comportamiento. En él explica que el usuario necesita de la motivación, habilidad y la incitación (triggers) para dar lugar a la conducta. Esto implica que los tres factores deben estar presentes en el mismo instante. Su trabajo se encuentra dentro de las Teorías del cambio de comportamiento, que estudian cómo se dan lugar los cambios en las conductas.

En el ámbito de los artefactos eléctricos y de consumo de recursos, Jelsma (2006) cuestiona la responsabilidad de los consumidores sobre los impactos en el medioambiente. En su argumento, explica que las incidencias negativas son la consecuencia de la forma en que los artefactos guían a las personas. En él, destaca la influencia de los objetos en la creación de hábitos, sean estos positivos o negativos. Consecuentemente, entiende a los diseñadores como un grupo que actúa moralmente a través de los productos. Según el autor, antes mencionado:

Los artefactos tienen una corresponsabilidad por la forma en que se desarrolla la acción y por los resultados. Si desperdiciamos energía o producimos residuos en acciones rutinarias como en las prácticas domésticas, eso tiene que ver con la forma en que los artefactos nos guían (p. 14).

A modo de conclusiones preliminares de este primer apartado, se entiende que si bien el DC cuenta con varios enfoques, sus objetivos se relacionan con influenciar las acciones indeseadas y fortalecer las positivas, a través de productos. Sus objetivos, se centran en reducir el consumo de recursos y evitar los impactos sociales negativos. Estos cambios se pueden lograr de diferentes maneras: de forma que facilite que los usuarios adopten un comportamiento deseado, a través de dificultades de acción para que el usuario lleve a cabo un comportamiento indeseado, promoviendo que las personas quieran un comportamiento deseado o provocando que los usuarios no quieran un comportamiento indeseado (Niedderer, 2014). A su vez el DC analiza el tipo de usuario que lleva a cabo la actividad teniendo en cuenta su psicografía. Los análisis de los usuarios incluyen tanto las actitudes, como las percepciones y las acciones reales llevadas a cabo, por medio de observaciones cuali-cuantitativas.

Dentro de las metodologías estudiadas en el proyecto de investigación Diseño con intención, motivación de conductas sustentables, mencionado previamente, se ha indagado específicamente en las metodologías utilizadas en Diseño con intención sustentable (Lockton, 2010), el desarrollo que en este estudio denominamos Diseño para un comportamiento 
sostenible (Lidman \& Renström, 2011), Diseño de comportamiento sostenible del consumidor (Olaya Rodríguez, 2018) y el Modelo de Intervención del Comportamiento (Tang, 2010).

El trabajo de Lockton (2010) se consolida a partir de la integración de múltiples herramientas y técnicas que permiten guiar el comportamiento, para alentar al usuario a adoptar la acción deseable de forma directa. Este conjunto de herramientas, adopta un enfoque funcional que considera la motivación (como una restricción interna) y el comportamiento habilitador y restrictivo (restricción externa a través del diseño). Este modelo concentra una diversidad de disciplinas de diseño (industrial, gráfico y arquitectónico entre otros) para la identificación de métodos que influyan en el comportamiento del usuario, lo que lo constituye en un modelo innovador. Formula el problema a través de ocho lentes, los cuales corresponden a una colección de patrones de conducta que se repiten en distintas disciplinas. Estos patrones son funcionales al diseñador como guía para traducir su investigación en una decisión de diseño encaminada a provocar un accionar a favor del medio ambiente. El autor reconoce dentro del total de la población, a personas con diferentes actitudes frente al cuidado del medioambiente y las divide bajo tres categorías para introducir diversos grados de control.

Tang analiza las herramientas de Lockton y concluye que se necesita un camino más directo para reducir los impactos de los artefactos, más que herramientas universales (Tang, 2010). En el modelo desarrollado por la autora se remarca la importancia de comprender las percepciones de los usuarios, a través de la investigación cualitativa-cuantitativa. Uno de los puntos centrales de su metodología es la observación y análisis del usuario como base para comprender los hábitos, intenciones, y elementos que explican la naturaleza de las acciones. Para ello combina estudios etnográficos con técnicas centradas en el usuario, las cuales permiten llegar a conocer las percepciones de éstos y obtener conclusiones sobre la forma de uso y a la vez del contexto en el cual está inserto. Esto da información valiosa al diseñador, quien puede introducir cambios directamente en aquellas acciones que están teniendo un efecto no deseado. En su tesis realiza un Modelo de Intervención del Comportamiento, en donde genera una conexión entre factores psicológicos (actitudes, afecto y hábitos) y estrategias de abordaje de diseño.

Las estrategias (que posteriormente utiliza Olaya Rodríguez) muestran diferentes orientaciones: Eco-información: El objetivo es hacer el consumo visible o que el usuario experimente físicamente la cantidad de energía necesaria para posibilitar el funcionamiento. Este tipo de productos da lugar a la reflexión. Eco-elección: Se alienta a los usuarios a que elijan entre diferentes opciones de uso entre las cuales se encuentran alternativas con un menor impacto. Eco-retroalimentación: El objetivo es informar a los usuarios sobre el estado operacional en el que se encuentra el artefacto para facilitar la toma de decisiones en tiempo real. Eco-estímulo: Inspira a usuarios a explorar un uso más sustentable a través de recompensas o sanciones. El objetivo es relacionar la conducta con la consecuencia. Eco-dirección: Direcciona a adoptar un hábito deseable a través de prescripciones y o restricciones. Intervención técnica: En este caso se restringen ciertas acciones automáticamente con la inserción de la tecnología. Evita la etapa en la que el usuario toma decisiones proponiendo soluciones automatizadas y Diseño inteligente, el cual induce a desarrollar acciones ambientalmente amigables exclusivamente a través de productos innovadores sin 
que el usuario se percate de ello. Estas estrategias son posibles caminos a seguir, aunque no están implementados masivamente en productos, tienen gran potencial en el camino de la sustentabilidad.

Siguiendo esta línea, Lidman y Renström (2011) desarrollan su propio esquema de intervención de diseño. Este se sistematiza en dos grandes grupos: según si el comportamiento de la persona de adapta al producto, o el diseño se adapta al comportamiento. A su vez, dentro del primer gran grupo encontramos subcategorías que explican los posibles caminos que puede desarrollar el proyectista: Enlighten (Iluminar): Motivar a las personas apelando e influenciando sus valores, conocimiento, actitudes y normas. Steer (Dirigir): guían al usuario haciendo que la elección evidente sea un comportamiento sostenible. Spur (Estimular): Alientan al usuario a realizar cierto comportamiento a través de recompensas. Force (Forzar): Impone un comportamiento a través de la limitación de la función. Y finalmente Match (Coincidir): El diseño se adapta al comportamiento de la persona, auto-reduciendo los impactos.

Finalmente, se analiza la metodología utilizada por Olaya Rodríguez (2018). La autora plantea intervenciones sostenibles que afectan directamente a la sociedad y al medio ambiente, a partir del desarrollo de productos y servicios que proyectan los diseñadores. De esta manera, son los proyectistas quienes deben asumir la responsabilidad de los resultados de sus productos. Debido a lo cual, la aplicación de estrategias de diseño sostenible resulta fundamental en pos de controlar los impactos consecuentes del consumo sobre los recursos naturales que explotan. Y dichos impactos se corresponden a los estilos de vida de la sociedad contemporánea, por esta razón es que la influencia en los comportamientos a través del diseño permite mejorar los hábitos hacia conductas sostenibles. Sin embargo, no todos los usuarios parten desde la misma base, y teniendo en cuenta la variedad de tipos de consumidores y los procesos del cerebro humano para llevar a cabo cualquier comportamiento (factores internos y externos que lo influyen, como motivación, capacidades y las pautas necesarias para concretarlo), es que la autora establece cuatro tipos de usuario sobre los cuales el diseño debe dirigir sus esfuerzos de formas diferentes, para generar los cambios de comportamiento. Con la meta de proyectar estrategias de diseño en función de la clasificación del usuario y basada en pautas psicológicas, Olaya Rodríguez (2018) determina cuatro tipos principales para el diseño sustentable: El perfil Entusiasta, el Preocupado, el Indeciso y el Irresponsable. Si bien la autora plantea la tipología de los usuarios para luego en base a eso aplicar el tipo de intervención de diseño para lograr el cambio de conducta, no expresa cuáles son los procedimientos que deben seguir los diseñadores para medir/identificar a cada usuario y ubicarlo en las mencionadas categorías pre-establecidas. A continuación, se realiza el estudio y análisis de la Psicología de la Conducta Sustentable, para luego desarrollar las vías a partir de las cuales identificar los usuarios que categoriza Olaya Rodriguez (2018).

Como conclusión preliminar, se observa que los autores comparten, en primer lugar, el análisis de los usuarios. Los hábitos, percepciones, emociones y características son recolectadas a través de encuestas y observación directa (no todos los enfoques llevan a cabo esta última). La observación es una herramienta de gran valor para establecer si existe correspondencia entre las percepciones de los usuarios y sus acciones. A su vez, permite observar dónde se encuentran las mayores fallas en el diseño. Por otro lado, concuerdan 
que a través de estas herramientas se pueden tipificar los usuarios según las características psicofísicas, que conducen las acciones responsables en menor o mayor medida. A su vez, para el DC la sustentabilidad en el uso, puede efectuarse forzando la conducta de la persona o permitiendo que el usuario reflexione. Dentro de esos dos puntos extremos encontramos una gran variedad que combina estrategias para promover una mejor conducta. Cabe mencionar que una parte de las estrategias sólo se propone concientizar sobre el impacto que generan los artefactos en el uso, otras proponen llevar a cabo una conducta sustentable automáticamente. Ahora bien, sin ser el objeto de este estudio, se identifican de forma general, algunos rasgos de comportamientos y variabilidad psicológica en tiempos de pandemia del COVID-19. En la mayoría de los países, como respuesta sanitaria ante la exponencial propagación del virus se ha adoptado el aislamiento comunitario. En Argentina, esta medida posee el carácter de aislamiento social, preventivo y obligatorio (Decreto 297/2020). Los efectos particulares de esta disposición se reflejan directamente en el comportamiento y por ende, en el uso de los artefactos en torno a ello. Hábitos de limpieza, permanencias prolongadas en los hogares y uso más frecuente de los objetos allí situados, dificultades en el abastecimiento de servicios urbanos, disminución (para gran parte de la población desempleada o que trabaja de manera informal) de ingresos familiares, entre otros; son algunas de las consecuencias particulares que se observan; sobre las cuales se debe contemplar un marco conceptual particular que se espera poder desarrollar en un próximo estudio. Por otro lado, desde el marco de la sustentabilidad que entiende los impactos ambientales de las prácticas sociales urbanas, se identifica en una disminución del consumo, mayor aprovechamiento de insumos hogareños y menor contaminación del aire por combustibles derivados del transporte (con una caída del 25\% de las emisiones de dióxido de carbono), entre otras modificaciones. Con respecto a los residuos por un lado, la gestión urbana de residuos se ve afectada por la precariedad laboral de muchos sectores; pero qué sucede dentro de los hogares en relación a los hábitos sustentables. Según Mongi (2020), hacer compost en casa es un hábito que sumó adeptos en cuarentena, el aislamiento hizo que más familias se iniciaran en la práctica, lo que implica reutilizar buena parte de sus desechos.

\section{La psicología de la Conducta Sustentable}

Los estudios conductuales generalmente son investigados por la psicología ambiental, la misma ha permitido desarrollar líneas teórico-prácticas respecto a los factores del entorno social que influyen en la conducta, reconociendo el papel de las normas establecidas, los contextos sociales, la educación y los valores éticos (Gifford, 2014). La gravedad de los problemas ambientales demanda a la disciplina psicológica que explore cómo los procesos cognitivos, emocionales y motivacionales propician la aparición de conductas sustentables (Schmuck \& Schultz, 2002). La investigación de las conductas protectoras del ambiente se ha dado mayoritariamente bajo el cobijo de la Psicología Ambiental. Esta área de la Psicología estudia las influencias recíprocas entre la conducta y el medio ambiente (Aragonés y Amérigo, 1998). Por su parte, la conducta sustentable es tema de análisis en 
un amplio número de investigaciones en psicología ambiental, orientados al estudio de los factores psicológicos que afectan y que son afectados por la interacción individuo-medio ambiente (Corral-Verdugo \& Queiroz Pinheiro, 2004).

Si bien, en la definición de sustentabilidad establecida por la World Commission on Environment and Development (1987) se entiende de forma implícita la relevancia del comportamiento ambiental responsable, éste no está definido en el documento. En consecuencia, a partir de la postulación de diversos autores, se procurará responder las siguientes preguntas: ¿Qué es la conducta sustentable? ¿Quiénes la definen? ¿Cómo se afianza esta concepción? ¿Cuáles son sus dimensiones?

Según Corral-Verdugo y Queiroz Pinheiro (2004) es posible diferenciar tres estadios en la investigación de la Conducta Sustentable. Durante la etapa Inicial (1960-1980) aparecen las primeras publicaciones especializadas en Psicología Ambiental (Environment $\&$ Behavior en 1969 y el Journal of Environmental Psychology, en 1980). Predominan las aproximaciones teóricas con base en el conductismo y a partir de ésta se elaboran modelos explicativos de la conducta de cuidado del ambiente. Las intervenciones que se utilizan con el objetivo de modificar los comportamientos anti-ambientales son los avisos o recordatorios que muestran cuál sería la conducta apropiada (Reid, Luyben, Rawers y Bailey, 1976; Katzev y Mishima, 1992), los reforzadores positivos para las acciones consideradas como pro-ecológicas (Burgess et al., 1971) y el castigo para las antiecológicas (Agras, Jacob y Ledebeck, 1980).

Durante el periodo Intermedio (1980-1990), se dejan de lado las aproximaciones con base en el conductismo para dar lugar a las aproximaciones de corte cognoscitivo. Los términos comportamiento proambiental, conducta pro-ecológica y conducta ambiental responsable, adquieren relevancia y empiezan a conformar el área de investigación de manera más distintiva. La Conducta ambiental, se entiende entonces, como el comportamiento intencional dirigido al cuidado del medio (Grob, 1990), centrando su atención en el entorno físico. Con respecto a las necesidades humanas, si bien las reconoce como importantes, quedan en segundo plano (Bonnes \& Bonaiuto, 2002).

El último y actual periodo (1990 - actualidad), coincide con los resultados de estudios que confirman los problemas ambientales tales como la disminución de los recursos que se consideraban ilimitados hasta entonces, como el agua (Brown y Flavin, 1999), al mismo tiempo que se confirma que el sobrecalentamiento global (Gardner, 2002). Durante esta etapa, los términos de conducta pro-ambiental, proecológica y ambiental comienzan a ser reemplazados por el concepto de conducta sustentable, el cual implícitamente se dirige a buscar no sólo el cuidado del entorno sino también a promover el bienestar humano en todos los rincones del planeta (Schmuck y Schultz, 2002; Bonnes y Bonaiuto, 2002; Pinheiro, 2002). La Conducta Sustentable es definida por Corral-Verdugo y Queiroz Pinheiro (2004) como aquel conjunto de acciones efectivas, deliberadas y anticipadas que resultan en la preservación de los recursos naturales, incluyendo la integridad de las especies animales y vegetales, así como en el bienestar individual y social de las generaciones humanas actuales y futuras. Estos autores definen la conducta sustentable a partir de rasgos, denominados dimensiones psicológicas de la conducta sustentable. Éstas, consisten en cinco características psicológicas: efectividad, deliberación, anticipación, solidaridad y austeridad. La efectividad implica responder de manera hábil ante requerimientos de cuidado 
del medio físico y social; la deliberación, significa que la conducta debe suceder teniendo el propósito específico de cuidar el ambiente y debe propiciar el bienestar humano y de otros organismos en el entorno. La anticipación, implica que aunque la conducta se realiza en la actualidad, el sujeto proyecta su acción al futuro, que es el tiempo al que se dirige su comportamiento presente. La solidaridad, se define como el conjunto de tendencias y acciones altruistas desplegadas en respuesta a la preocupación por los demás. Finalmente, la austeridad, plantea la necesidad de llevar un estilo de vida en el que el consumo de bienes y recursos naturales se limite a lo indispensable.

En 2011, Corral-Verdugo realiza una re-categorización de las dimensiones psicológicas de la conducta sustentable. Las identifica como comportamientos pro-ecológicos, altruistas, frugales y equitativos. Las conductas pro-ecológicas, las define como un conjunto de acciones intencionales y efectivas que tienen como finalidad conservar el ambiente natural; constituye una de las dimensiones fundamentales en la conformación de la conducta sustentable. El altruismo expresa la disposición de las personas para actuar a favor de sus semejantes de manera desinteresada, sin esperar ningún tipo de gratificación a cambio. Numerosas investigaciones han demostrado que los sujetos altruistas que se involucran en acciones de ayuda a otros individuos también se orientan hacia la protección del ambiente natural (Corral Verdugo, 2010). La frugalidad hace referencia a un estilo de vida sencillo, que establece un equilibrio en el plano social entre clases, así como en el individuo entre sus necesidades y medios. Finalmente la equidad es el supuesto de que los individuos merecen un tratamiento de igualdad y justo más allá de la variedad de las características sociales, biológicas y demográficas que los definen. Por otra parte, el autor menciona que la intolerancia, el sexismo y el establecimiento de sistemas sociales jerárquicos son opuestos a la idea de equidad e influyen de manera negativa en el comportamiento pro-ambiental.

\section{Determinación de las relaciones existentes en la categorización que realiza Olaya Rodríguez (2018) y las dimensiones de la conducta sustentable}

En base a las sub-escalas de conducta sustentable que emplea la psicología de la conducta ambiental, se identifican los perfiles de usuario que propone Olaya Rodríguez (2018) en su trabajo. Dichas sub-escalas se componen aproximadamente de diez variables, que representan las afirmaciones que el encuestado debe calificar según una escala tipo Likert, con cinco opciones de respuesta (de 0 a 5 ó de muy en desacuerdo con totalmente de acuerdo); las mismas se denominan como las Conductas pro-ecológicas de Kaiser (1998) adaptada por Tapia, Fraijo, Corral, Gutiérrez y Tirado (2006), como un conjunto de acciones intencionales y efectivas que resultan en la conservación del ambiente natural con el propósito de conservar el capital natural y evitar su deterioro, por ejemplo el hábito de reuso o reciclaje; a partir de ahora se refiera como escala Pro-ecológica en el resto del documento. El Altruismo (Tapia et al, 2006), valora las acciones que se dirigen a la atención de las necesidades de otras personas; en general, representa a personas que se sienten motivadas por el deseo de mejorar el bienestar del prójimo. Por último, la Anticipación o propensión al 
futuro (Tapia et al, 2006), que considera las necesidades de las futuras generaciones, aparte de las de las presentes.

A partir de los resultados que reconocen a cada tipo de usuario, se los identifica según las cuatro categorías que describe Olaya Rodriguez (2018): los Entusiastas, caracterizados por su predisposición a comprar productos sostenibles y estar involucrados con los mismos, por lo que principalmente se los identifica con comportamientos pro-ambientales, por lo que se esperan altos valores en la sub-escala adaptada por Tapia, Fraijo, Corral, Gutiérrez y Tirado (2006). Los Irresponsables, opuestos conductualmente a los primeros, no se encuentran predispuestos, ni interesados, ni involucrados con el consumo sostenible, por lo cual se los detecta cuando se miden bajos valores en las sub-escalas de conducta Proecológica (Kaiser, 1998) adaptada por Tapia, Fraijo, Corral, Gutiérrez y Tirado (2006) y de Altruismo (Tapia et al, 2006). Para los Preocupados, caracterizados por su responsabilidad expresada en comportamientos pro ambientales y el bienestar de las futuras generaciones se los identifica al medir valores medios con tendencia a altos para la sub-escala Proecológica de Kaiser (1998) adaptada por Tapia, Fraijo, Corral, Gutiérrez y Tirado (2006) y valores altos en la sub-escala de Anticipación o propensión al futuro (Tapia et al, 2006). Finalmente, los Indecisos, se definen como aquellos que se sienten responsables de los problemas ambientales pero están confundidos acerca de si sus acciones pueden cambiar su situación, se espera que en la Pro-ecológica (Kaiser, 1998) adaptada por Tapia, Fraijo, Corral, Gutiérrez y Tirado (2006) obtengan valores medios y en la sub-escala de Altruismo (Tapia et al, 2006) valores bajos (Retamozo \& Clinckspoor, 2019).

A continuación, en la Figura 1, se relacionan de forma explícita cada tipo de usuario de acuerdo a las sub-escalas que se relevan en el momento de realizar las encuestas. De esta manera, los diseñadores pueden claramente comprender en qué categoría incluir a cada usuario de acuerdo a los resultados recabados.

\begin{tabular}{|c|c|c|c|c|}
\hline \multirow{2}{*}{$\begin{array}{l}\text { Tipo de usuario } \\
\text { (Olaya Rodríguez, 2018) }\end{array}$} & \multirow{2}{*}{ Definición } & \multicolumn{3}{|c|}{$\begin{array}{c}\text { Valores esperados en las diferentes } \\
\text { sub-escalas }\end{array}$} \\
\hline & & $\begin{array}{l}\text { Pro-ecologica } \\
\text { (Kaiser, 1998) }\end{array}$ & $\begin{array}{c}\text { Altruismo } \\
\text { (Tapia et al 2006) }\end{array}$ & $\begin{array}{l}\text { Prop. al futuro } \\
\text { (Anticipación) } \\
\text { (Tapia et al 2006) }\end{array}$ \\
\hline Entusiasta & $\begin{array}{l}\text { Opinan que es muy positivo y significativo comprar } \\
\text { productos sostenibles y están involucrados con ellos. } \\
\text { Este segmento de consumidores ya ha realizado } \\
\text { (quizás continua realizando) comportamientos pro- } \\
\text { ambientales. } \\
\text { Tienden a ser sensibles a los temas ambientales. } \\
\text { Estos consumidores creen que sus elecciones } \\
\text { significan mucho para el medio ambiente. }\end{array}$ & & & \\
\hline \multirow[t]{2}{*}{ Irresponsable } & $\begin{array}{l}\text { No tienen una actitud positiva hacia los productos } \\
\text { sostenibles, ni tienen la intención de comprarlos, poco } \\
\text { involucrados con el consumo sostenible. No les } \\
\text { interesa realizar acciones pro ambientales. }\end{array}$ & & & \\
\hline & Son egocéntricos (Opuesto aAltruista) & & & \\
\hline
\end{tabular}

continúa $>>$ 


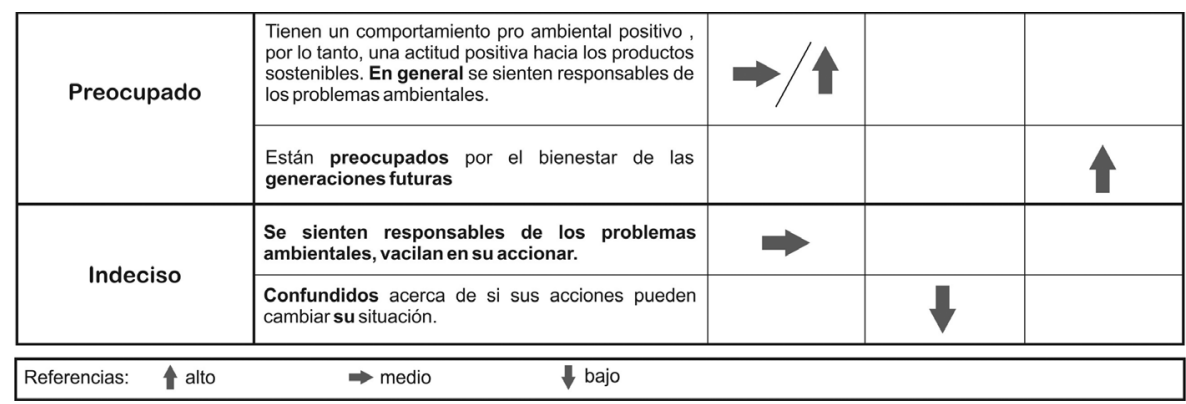

Figura 1. Cruzamiento de datos entre la clasificación de usuarios que realiza Olaya Rodríguez y las subescalas de detección de conducta sustentable. Fuente: Elaboración propia.

\section{Reflexiones finales}

La función del diseño conlleva un impacto esperado sobre el uso del bien cultural que materializa; de esta manera, se entiende a los artefactos como poseedores de cualidades intrínsecas a su proceso de proyección que determina la manera en la que los consumidores los perciben, aprecian y emplean. A partir de este postulado es que se abordaron las preguntas que guiaron esta investigación, respecto a cómo pueden los y las diseñadores identificar a los distintos usuarios, con qué parámetros los clasifican y cómo miden sus conductas para establecer qué tipo de usuario resultan, en pos de esclarecer de qué manera se vincula la tipificación con las herramientas que pueden emplearse para medir a cada usuario.

La tipificación que realiza Olaya Rodriguez (2018), como se ha explicado anteriormente, se sostiene sobre pautas psicológicas, las cuales se determinan a partir de llevar a cabo una encuesta sobre la conducta sustentable de los usuarios. Por este motivo, las herramientas que brinda la Psicología de la Conducta sustentable, son un complemento, que permite identificar a los distintos usuarios a través de las sub-escalas de conducta sustentable que utiliza Corral Verdugo $(2004,2010)$ para medir las características psicológicas en las personas. Éstas permiten detectar patrones a partir de llevar a cabo una encuesta a los sujetos objetivo. En este trabajo se han tomado como parámetros de medición tres de las cinco sub-escalas que propone Corral Verdugo en su trabajo $(2004,2010)$ : la sub-escala de conducta Pro-ecológica de Kaiser (1998) adaptada por Tapia, Fraijo, Corral, Gutiérrez y Tirado (2006), el Altruismo (Tapia et al, 2006) y Anticipación o Propensión al Futuro (Tapia et al, 2006). Las encuestas, por su parte deben ser lo suficientemente rigurosas para recabar buena cantidad y calidad de datos que permitan determinar el perfil del usuario. En este sentido, se han sistematizado algunos patrones de comportamientos de usuarios asociados a perfiles predispuestos, en distintos grados y los que no, para con el medio ambiente. Para considerar efectiva esta correspondencia diseñador-usuario, resultó necesario 
conocer arquetipos, que distintos tipos de usuario comparten y manifiestan ante estímulos que velan por la sustentabilidad. Estas generalizaciones de los fenómenos sociales se ven representadas en las actitudes y las conductas que adoptan los usuarios, a lo largo del tiempo y en contextos culturales específicos.

Como se evidencia en este trabajo, las definiciones han evolucionado en la consolidación de la corriente teórica estudiada; por ejemplo, la denominada conducta proambiental, la cual solo considera acciones protectoras del medio ambiente, se ha transformado en el término conducta sustentable. Esta última, incorpora los cuatro niveles de sustentabilidad: económico, político, ambiental y social. En este sentido, autores contemporáneos, como Corral-Verdugo y Queiroz Pinheiro, evalúan la conducta sustentable de los individuos basándose en dimensiones psicológicas de la sustentabilidad. Conocer estas características permite al proyectista, comprender previamente las tendencias del fenómeno social a ser valoradas; ya que muchas veces los objetivos propuestos en el diseño de los productos no se terminan concretando en la etapa de uso del artefacto.

En conclusión, se considera que para que el diseño ambientalmente sostenible se consolide de manera efectiva debe tener en cuenta la evaluación de las reacciones de los usuarios ante diferentes motivaciones. De modo que permita a estudiantes y profesionales, reflexionar sobre alternativas de diseño que refuercen la intención con la que crean sus productos, al reconocer y evaluar diferentes predisposiciones que pueden sostener los usuarios ante estímulos que aspiren a comportamientos sustentables.

Se considera que el impacto de este tipo de estudios, independientemente de mejorar la práctica del diseño para el cambio de comportamiento, trasciende el escenario académico, dado que posibilita abordar problemas sociales y ambientales, reuniendo conocimiento de diferentes disciplinas y estableciendo paralelos que posibilitan la transposición de conceptos.

Por último, en relación a los efectos de la pandemia sobre los comportamientos, resulta emergente la producción de análisis que permitan al diseño orientar sus esfuerzos hacia mejorar la degradación ambiental derivada de los hábitos sociales urbanos. Se entiende que también se abren nuevas posibilidades a las cuales adaptarse, para pensar nuevos modelos de producción y de acciones en relación al rol del Diseño Conductual en tiempos de COVID-19 y pos pandemia. A partir de este trabajo, se espera desarrollar en el futuro, un análisis de las variables y condicionamientos sustentables alterados en situaciones de confinamiento social general y acondicionar las cualidades estructurales de un entorno socio ambiental específico. De esta manera, se abre la puerta para un próximo trabajo en el cual se aborde la pregunta de cómo influye el Diseño en contextos extremos como el actual. Para dicho estudio se debe desarrollar un marco teórico particular para tal caso, desde una mirada global que pueda medirse en sus efectos locales particulares.

\section{Listado de referencias bibliográficas}

Agras, W. S.; Jacob, R. G. y Ledebeck, M. (1980). The California drought: A quasi experimental analysis of social policy. Journal of Applied Behavior Analysis, 13, 561-570. 
Akrich, M. (1992). The De-Scription of technical objects. Shaping Technology/ Building Society: studies in sociotechnical change. MIT Press, Cambridge, pp 205-224.

Aragonés, J. I. \& Amérigo, M. (1998). Psicología ambiental. Aspectos conceptuales y metodológicos. Psicología ambiental, 21-41.

BBC (2 de marzo de 2020). Coronavirus: las imágenes que muestran la sorprendente caída de la contaminación del aire en China desde el inicio de la crisis. BBC Recuperado de: https://www.bbc.com/mundo/noticias-51713162

Bonnes, M. \& Bonaiuto, M. (2002). Environmental psychology: From spatial-physical environment to sustainable development. En Handbook of environmental psychology, 28-54.

Brown, L. R. y Flavin, C. (1999). A new economy for a new century. In L. Starke (Ed.), State of the World. A Worldwatch Institute Report on Progress Toward a Sustainable Society. New York: W.W. Norton \& Company.

Burgess, R. L.; Clark, R. N. y Hendee, J. C. (1971). An experimental analysis of antilitter procedures. Journal of Applied Behavior Analysis, 4, 71-75.

Caballero Quiroz, J. A. y Mercado González O. (2018). Affordance y Diseño. UAM, Unidad Cuajimalpa, División de Ciencias de la Comunicación y Diseño (pp. 8-13). México.

Cone, J. D. y Hayes, S. C. (1980). Environmental problems. Behavioral solutions. Monterey, CA: Brooks Cole.

Corral-Verdugo, V. (2011). Glosario de términos psicoambientales. En Diccionario temático de psicología (pp. 67-78). D.F. México: Trillas.

Corral, V. (2010). Psicología de la sustentabilidad. Un análisis de lo que nos hace pro ecológicos y pro sociales. México: Trillas

Corral-Verdugo, V. \& Pinheiro, J. (2004). Aproximaciones al estudio de la conducta sustentable. Medio ambiente y comportamiento humano, 5(1), 1-26.

Environmental Change Institute. (2005). 40\% House Report. Oxford: Oxford University.

Fogg, B. J. (2003). Persuasive Technology: Using Computers to Change What We Think and Do. San Francisco: Morgan Kaufmann.

Fogg, B. J. (2009). A behavior model for persuasive design. In Proceedings of the 4th International Conference on Persuasive Technology (Persuasive '09). Association for Computing Machinery, New York, NY, USA, Article 40, 1-7.

Gardner, G. (2002). The challenge for Johannesburg: Creating a more secure world. En L. Starke (Ed.), State of the World 2002. A Worldwatch Institute Report on Progress Toward a Sustainable Society. Nueva York: W.W. Norton \& Company.

Gifford, R. (2014). Environmental psychology matters. En Annual review of psychology, 65, 541-579. Canada: University of Victoria.

Grob, A. (1990). Meinungen im Umweltbereich und umwelgerechtes Verhalten. Ein psychologisches Ursachennetzmodell. Tesis Doctoral. Suiza: Universidad de Berna.

Jelsma, J. (2006). Designing 'moralized' products. In: Verbeek PP., Slob A. (eds) User Behavior and Technology Development. Dordrecht: Springer.

Kaiser, F. G., \& Shimoda, T. A. (1999). Responsibility as a predictor of ecological behaviour. Journal of Environmental Psychology, 19(3), 243-253.

Katzev, R. \& Mishima, H. R. (1992). The use of posted feedback to promote recycling. En Psychological Reports, 71(1), 259-264. 
Latouche, S. (2012). La sociedad de la abundancia frugal. Contrasentidos y controversias del Decrecimiento. Barcelona: Icaria.

Lidman, K. \& Renström, S. (2011). How to design for sustainable behaviour? A review of design strategies and an empirical study of four product concepts. Chalmers: Chalmers University of Technology.

Lilley, D. (2007). Designing for behavioural change: reducing the social impacts of product use through design. Loughborough: Loughborough University. Thesis. https://hdl. handle.net/2134/8092

Lockton, D. (2010). Design with intent: 101 patterns for influencing behaviour through design. Windsor: Equifine.

Lockton, D.; Harrison, D. \& Stanton, N. A. (2012). Models of the user: designers perspectives on influencing sustainable behaviour. En Journal of Design Research Vol.10 No.1/2, pp.7-27.

Manzini, E. \& Meroni, A. (2007). Emerging user demands for sustainable solutions, EMUDE. En Design Research Now (pp. 157-179). Basel: Birkhäuser.

Martínez Alier, J. (2009). El ecologismo de los pobres: conflictos ambientales y lenguajes de valoración (No. Sirsi) i9788474267433). Barcelona: Icaria.

Myllyvirta, L. (2020). Analysis: Coronavirus temporarily reduced China's CO2 emissions by a quarter. Carbon Brief. Retrieved March 25, 2020, from https://www.carbonbrief. org/analysis-coronavirus-has-temporarily-reduced-chinas-co2-emissions-by-a-quarter

Mongi, C. (08 de mayo de 2020). Hacer compost en casa: un hábito que sumó adeptos en cuarentena. En La Voz. Recuperado de: https://www.lavoz.com.ar/ciudadanos/hacercompost-en-casa-un-habito-que-sumo-adeptos-en-cuarentena

Niedderer, K.; Mackrill, J.; Clune, S.; Lockton, D.; Ludden, G.; Morris, A.; Caín, R.; Gardiner, E.; Gutteridge, R.; Evans, M. y Hekkert, P. (2014). Creating sustainable innovation through design for behaviour change: full project report. Wolverhampton: University of Wolverhampton. Project Partners \& AHRC, 126pp.

Norman, D. A. (1988). The psychology of everyday things. New York: Basic books.

Olaya Rodriguez, A. (2018). Make Them Behave Sustainably: Applying strategies to design sustainable behaviour. USA: Ed. Amazon.

Pinheiro, J. (2002). Apego ao futuro: escala temporal e sustentabilidade em psicologia ambiental. En V. Corral-Verdugo (Ed.). Conductas protectoras del Ambiente. México: CONACyT-UniSon.

Retamozo, E. y Clinckspoor, G. (2019). Tipificación de las conductas ambientales para el diseño con intención sustentable. XVII Edición de la Semana Nacional de la Ciencia y la Tecnología. Mar del Plata - Argentina.

Reid, D. H.; Luyben, P. D.; Rawers, R. J. \& Bailey, J. S. (1976). Newspaper recycling behavior: The effects of prompting and proximity of containers. En Environment and Behavior, $8(3), 471-482$.

Schmuck, P. \& Schultz, P.W. (2002). Sustainable development as a challenge for psychology. En Psychology of sustainable development (pp. 3-17). Boston, MA: Springer.

Tang, T. (2010). Towards sustainable use: design behaviour intervention to reduce household environment impact. London: Loughborough University. 
Tapia, C.; Fraijo, B.; Corral, V.; Gutiérrez, C. \& Tirado, H. (2006). Validación de una escala de orientación hacia la sustentabilidad. En B. Fraijo, S. Echeverría y C. Tapia (Eds.), Desierto $y$ mar. Estudios sociales en Sonora. Sonora: Instituto Tecnológico de Sonora.

World Commission on Environment and Development (WCED), S. W. S. (1987). World commission on environment and development. En Our common future, 17, 1-91.

https://www.argentina.gob.ar/coronavirus/aislamiento (Consultado el 26/05/2020)

https:/www.who.int/es/emergencies/diseases/novel-coronavirus-2019/advice-for-public/qa-coronaviruses (Consultado el 26/05/2020)

\begin{abstract}
Design artifacts are considered to hold qualities intrinsic to their projection process which determine the way in which users perceive, appreciate, and employ the objects. For the environmentally sustainable design to consolidate, the evaluation of the subjects' reactions under different motivations must be consolidated. The aim of this essay is to base, through theoretical-methodological terms, the conceptual definitions of the users which are typified within the Design of sustainable behavior, and which are studied in the context of environmental psychology by conceiving the behavior from different dimensions in order to identify different environmental profiles. Finally, the work averages the deepening of the objective towards a future study, in which the problem is addressed in an extraordinary context, such as a worldwide pandemic.
\end{abstract}

Keywords: Sustainable Design - Behavioral design - Sustainable habits - Environmental psychology - Types of users.

Resumo: Os artefatos são entendidos como possuindo qualidades intrínsecas ao processo de design, que determinam a maneira pela qual os usuários os percebem, apreciam e os utilizam. Para que o desenho ambientalmente sustentável seja consolidado, deve-se considerar a avaliação das reações dos sujeitos a diferentes motivações.

O objetivo deste ensaio é apoiar, através de termos teórico-metodológicos, as definições conceituais dos usuários tipificados no Design de Comportamento Sustentável, estudados por autores especializados em psicologia ambiental que concebem comportamentos de diferentes dimensões, para identificar os diferentes perfis de Meio Ambiente

Finalmente, o trabalho calcula a média do aprofundamento do objetivo em direção a um estudo futuro, no qual o problema é abordado no contexto de situações extraordinárias, como uma pandemia mundial.

Palavras chave: Design sustentável - Design comportamental - Hábitos sustentáveis - Psicologia ambiental - Tipos de usuários.

[Las traducciones de los abstracts fueron supervisadas por el autor de cada artículo] 